\title{
On the Design of Online Scheduling Algorithms for Advance Reservations and QoS in Grids
}

\author{
Claris Castillo, George N. Rouskas, Khaled Harfoush \\ Department of Computer Science \\ North Carolina State University \\ Raleigh, NC 27695 \\ Email: \{ccastil,kaharfou,rouskas\}@ncsu.edu
}

\begin{abstract}
We consider the problem of providing QoS guarantees to Grid users through advance reservation of resources. Advance reservation mechanisms provide the ability to allocate resources to users based on agreed-upon QoS requirements and increase the predictability of a Grid system, yet incorporating such mechanisms into current Grid environments has proven to be a challenging task due to the resulting resource fragmentation. We use concepts from computational geometry to present a framework for tackling the resource fragmentation, and for formulating a suite of scheduling strategies. We also develop efficient implementations of the scheduling algorithms that scale to large Grids. We conduct a comprehensive performance evaluation study using simulation, and we present numerical results to demonstrate that our strategies perform well across several metrics that reflect both user- and system-specific goals. Our main contribution is a timely, practical, and efficient solution to the problem of scheduling resources in emerging on-demand computing environments.
\end{abstract}

\section{INTRODUCTION}

Grids have emerged as an essential infrastructure for resource-intensive scientific and commercial applications [12], [15]. Grid technology enables the sharing and dynamic allocation of distributed, high-performance computational resources while minimizing the associated ownership and operating costs; it also facilitates access to such resources and promotes flexibility and collaboration among diverse organizations. More recently, the concept of on-demand computing [5], [16] has emerged as a viable model in which a wide range of finer grain commercial, business, and scientific applications would tap into the Grid resources on an as-needed basis, extending the reach and utility of Grid computing far beyond its current user base to society as a whole; for instance, Sun Microsystems recently started offering the Sun Grid compute utility [18] and more such service offerings are expected in the near future. This vision of computing as utility is expected to change not only the way scientists and businesses work, but also the way they think about computing resources. However, its realization depends on the development of sophisticated resource management systems capable of allocating resources to users based on agreed upon quality of service (QoS) requirements [1], while satisfying certain system level objectives (e.g., high utilization, economic constraints, etc.) [3], [4].

Scheduling and management of Grid resources is an area of ongoing research and development. Several open source

This work was partially supported by NSF grants CAREER ANIR-0347226 and CNS-0434975.

1-4244-0910-1/07/\$20.00 (C)2007 IEEE. or proprietary schedulers have been developed for clusters of servers, including Maui [13], [14], portable batch system (PBS) [2], and load sharing facility (LSF) [9]; they typically run in batch mode, can be customized to specific policies, and attempt to balance the load among the various servers. However, the primary objective of most existing approaches is to improve overall system performance (e.g., utilization), while the QoS experienced by Grid users is at best of secondary consideration [15]. For instance, batch systems typically allocate resources to jobs as they become available, without consideration of applications that need to obtain results within a strict deadline [1]. In general, the schedulers process jobs in order of priority, which is determined based on job attributes such as job class and time in queue [13], [14], but also employ backfilling operations, i.e., run jobs out of order, to make better use of the available resources. Unfortunately, backfilling often interferes with the ability of the system to provide QoS guarantees, as in its attempt to improve utilization it may bypass the job priorities set by the system administrator [14].

Advance reservation of resources is one mechanism that Grid providers may employ in order to offer specific QoS guarantees to application users. Advance reservation, i.e., the ability of the scheduler to guarantee the availability of resources at a particular time in the future, increases the predictability of the system and it has been an area of interest [1], [11], [12], [15], [19]-[21] in the Grid community. Although some schedulers, including Maui [13], provide some sort of advance reservation mechanisms, existing approaches to making reservations in the future lack sophistication, are expensive, and do not scale well. This lack of scalability is due primarily to two factors. First, as the number of resources in the Grid increases, the overhead of maintaining and updating the set of advance reservations can be significant, especially if appropriate attention is not paid to the design of the relevant data structures. Second, making advance reservations tends to fragment the available resources. If this fragmentation is not taken into account by the scheduling algorithm, the result will be poor utilization and high job rejection rate; on the other hand, algorithms which attempt to utilize the fragmented capacity but are not properly designed will suffer from unacceptably high running times as the number of resources increases. For these reasons, incorporating QoS mechanisms into current Grid environments has proven to be a challenging task [1], [12]. In practice, most systems tackle the complexity by limiting both the pool of resources available for advance reservation and the number of users with permission to request 
reservations.

We believe that the ability to offer and guarantee QoS to users is of utmost importance to Grid providers. Without QoS guarantees, users may be reluctant to pay for Grid services or contribute resources to Grids, hindering further development of the Grid model and limiting its economic significance. Mechanisms for support of QoS also enable service providers to differentiate themselves by offering an optimized menu of services. Therefore, in this paper we present a framework for designing effective and efficient scheduling algorithms that employ advance reservations to guarantee QoS to users. Specifically, we consider an environment where users submit jobs dynamically, and these jobs may start at a future time and must be completed within a certain deadline. Using concepts from computational geometry [10], we show how to manage efficiently the fragmentation of resources due to advance reservations by maintaining an appropriate set of balanced search trees. We also present a set of scheduling strategies for making advance reservations. Each strategy corresponds to a different optimization objective, and requires that the information on the advance reservations be organized and maintained in a slightly different variant of the search tree structure. Our algorithms scale to large Grid systems, and simulation results demonstrate that they perform well across several performance metrics that reflect both user- and systemspecific goals.

The rest of the paper is organized as follows. In Section II we describe the online scheduling problem we study in this work, and in Section III we present a framework for reasoning about advance reservations that borrows ideas from computational geometry; we also describe a suite of scheduling strategies that arise naturally within the framework. In Section IV we provide additional details on the implementation of the scheduling algorithms and of the data structures related to managing the fragmentation of resources. In Section $\mathrm{V}$ we present simulation results to evaluate the various strategies in terms of several performance metrics, and we conclude the paper in Section VI.

\section{PROBLEM DESCRIPTION}

Consider a scheduler $\mathcal{S}$ for a Grid with $n$ servers which may be geographically distributed in a network. We make the assumption that all servers are identical in terms of their processing capacity $C$; extending the algorithms we present here to non-identical resources is the topic of ongoing research in our group. A user with job $j$ requiring service submits a request to the scheduler. The request is characterized by a three-parameter tuple $\left(r_{j}, l_{j}, d_{j}\right)$, where:

1) $r_{j}$ is the ready time of the job, i.e., the earliest the job can be made available to the grid for processing;

2) $l_{j}$ is the length of the job, i.e, the amount of work the job requires; and

3) $d_{j}\left(\geq r_{j}+l_{j}\right)$ is the deadline of the job, i.e., the latest time by which the job can be completed.

The deadline is a measure of the quality of service required by the user. We assume that deadlines are hard, in that a user receives utility only if the job completes service by its deadline. Therefore if $\mathcal{S}$ determines that the deadline cannot be met, it drops the job and notifies its user accordingly.


Fig. 1. (a) Advance reservations in a 2-server system: jobs scheduled and idle periods, (b) equivalent geometric representation of the schedule: idle periods as points in the plane

We consider the online scheduling problem whereby users submit service requests to $\mathcal{S}$ at random instants. We assume that $\mathcal{S}$ maintains a schedule which records, for each server $i$, the time periods in the future during which the server is reserved for jobs that have already been accepted to the system. In essence, this schedule represents the set of advance reservations that have been made, and it guarantees that server resources will be available to the accepted jobs at specific future times. Figure 1(a) shows an example schedule for a 2 -server system. The schedule shows that at the current time (i.e., time $t=0$ in the figure), there are three jobs scheduled for server 1: the job currently in service which will end at time $t_{1}$, job $A$ which has reserved the server from time $t_{3}$ to $t_{5}$, and job $D$ which has reserved the server from time $t_{9}$ to $t_{11}$; similarly, three jobs have been scheduled for server 2 . The figure also shows a service request for scheduling a new job $j$ with ready time $r_{j}=t_{6}$ and length $l_{j}=t_{8}-t_{6}$.

When a service request $\left(r_{j}, l_{j}, d_{j}\right)$ for a new job $j$ arrives, $\mathcal{S}$ immediately runs an algorithm to determine whether it is feasible to schedule the job so as to meet its deadline. If so, then $\mathcal{S}$ uses a set of criteria to select one of the (possibly multiple) servers who can handle this job, updates its schedule, and returns a reference to this server to the user; otherwise, the job is dropped. The scheduling decision impacts the performance perceived by users as reflected by the fraction of jobs meeting (or missing) their deadlines and the turnaround times of the jobs. It also impacts the overall system performance as reflected by the system utilization, which is a measure of how well the overall service capacity of the system is used. The challenge, therefore, is to develop efficient online scheduling algorithms that minimize the fraction of dropped jobs while maximizing utilization.

Several variants of this scheduling problem with advanced reservations and/or deadlines have been studied in multiprocessor and Grid systems [6], [7], [19], [20], [22]. However, the heuristic solution approaches that have been proposed may not scale well and may not utilize the available system capacity efficiently [1], [12]. In the next section, we present a new framework for developing efficient algorithms for this problem taking into account a range of optimization criteria.

Before we proceed to address the general scheduling problem, let us consider a restricted version in which jobs must be scheduled as soon as they are ready. In this case, deadlines are immediate (i.e., $d_{j}=r_{j}+l_{j}$ ), and we refer to this problem as resource scheduling with immediate deadlines. One straightforward approach for tackling this problem is 
for the scheduler $\mathcal{S}$ to keep track of the completion time of each server, defined as the latest time at which the server becomes free based on the existing advanced reservations. The scheduler then assigns an arriving job to the server with the latest completion time that is earlier than the ready time of the new job. This latest available completion time (LACT) algorithm takes time $O(\log n)$ to schedule a job. However, it can be inefficient in terms of both capacity utilization and job drop rate, as it does not consider the idle periods created at each server between the times reserved for jobs whose requests were submitted earlier. For instance, in the scenario shown in Figure 1(a) for a 2-server system, the completion time for server 1 is $t_{11}$ (the service completion time of job $D)$, while the completion time for server 2 is $t_{10}$. Therefore, the LACT algorithm will reject the service request for the new job with arrival time $t_{6}<t_{10}<t_{11}$, although the job can be accommodated on server 1 within the idle period $Y$ created between jobs $A$ and $D$.

An algorithm that considers the idle periods when making decisions was developed in [23] in the context of scheduling bursts in optical burst switched networks. The algorithm uses concepts from computational geometry [10] to represent the time intervals corresponding to idle periods as points in a plane, as illustrated in Figure 1(b). Since the ending time of an idle period must be greater than its starting time, all points will always be above the diagonal in Figure 1(b). Then, the problem of finding a feasible idle period for scheduling a new job (also represented as a point $P$ in the plane) is equivalent to finding a point that completely contains $^{1}$ point $P$. In Figure 1(b), it is seen that point $Y$ completely contains the point corresponding to the new job, thus the latter can be scheduled within idle period $Y$ on server 1 . By maintaining a balanced priority search tree data structure [17] containing all the idle periods on all servers, finding an idle period for a new job, or determining that one does not exist, takes time $O(\log K)$, where $K$ is the number of idle periods. Updating the data structure to add new idle periods (created when a new job is scheduled) or remove ones in the data structure (as time advances), also takes time $O(\log K)$. The value of $K$, however, can be significantly larger than the number $n$ of servers, and we have found that its value increases rapidly with the offered load of jobs; in other words, in moderately to highly loaded systems, in which it is important to make scheduling decisions quickly, the running time of the algorithm is longer.

\section{Scheduling With General Job DeAdlines}

We now present a general framework that provides new insight into the problem of online scheduling with advance reservations in Grid environments. Our approach extends previous work in three directions: (1) it allows for general job deadlines (i.e., the deadline of a job $j$ may take any value $d_{j} \geq r_{j}+l_{j}, \forall j$ ); (2) it provides the foundation for formulating a range of scheduling strategies based on a variety of optimization criteria; and (3) it leads to highly efficient algorithms for these strategies.

\footnotetext{
${ }^{1}$ We say that point $x=\left(x_{1}, x_{2}\right)$ completely contains point $y=\left(y_{1}, y_{2}\right)$ iff $x_{1} \leq y_{1}$ and $x_{2} \geq y_{2}$.
}

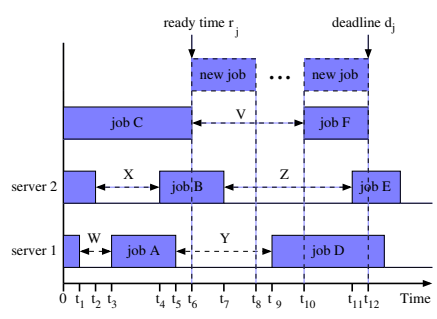

(a)

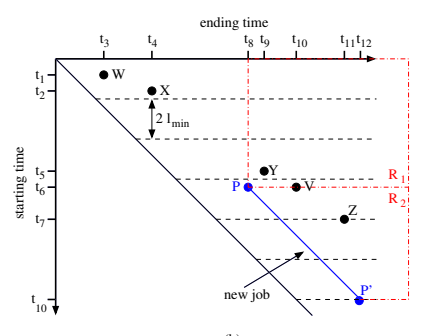

(b)
Fig. 2. (a) Jobs scheduled and idle periods in a 3-server system, (b) idle periods as points in the plane, plane partitioned into strips of width $2 \times l_{\text {min }}$, and feasible regions $R_{1}, R_{2}$ for the new job

Let us return to the representation of idle periods as points in the plane that we illustrated in Figure 1. Assuming that the current time $t=0$, Figure 2(a) shows the current schedule of advance reservations for a 3-server system, along with a request to schedule a new job $j$ with the tuple $\left(r_{j}=t_{6}, l_{j}=\right.$ $\left.t_{8}-t_{6}, d_{j}=t_{12}\right)$. Figure $2(\mathrm{~b})$ is the geometric representation of this schedule. The fact that job $j$ has a general deadline is represented in Figure 2(b) by the line segment between points $P$ and $P^{\prime}$, where point $P=\left(r_{j}, r_{j}+l_{j}\right)$ (respectively, $\left.P^{\prime}=\left(d_{j}-l_{j}, d_{j}\right)\right)$ corresponds to the earliest (respectively, latest) possible pair of starting and ending times for this job. Consequently, the scheduler may select any point on this line segment as the starting/ending times of the job, as long as there is an idle period completely containing this point.

Consider the new job $j$ and its geometric representation in the plane, as shown in Figure 2(b). The feasible region of job $j$ refers to the part of the plane where all idle periods that can accommodate this job may lie. The feasible region is the part of the plane above and to the right of the line segment between $P$ and $P^{\prime}$, since only any idle periods in that region will fully contain some point of the line segment. The feasible region can be partitioned into two subregions, $R_{1}$ and $R_{2}$, as in Figure 2(b). Any idle period lying in $R_{1}$ (e.g., idle periods $Y$ an $V$ in the figure) starts at or before the new job's ready time $r_{j}$ ( $=t_{6}$ in the figure), and ends after the earliest time the job can be completed ( $=t_{8}$ in the figure). Therefore, any idle period in this region can accommodate the new job without delaying its execution, i.e., the job can start execution at its ready time $r_{j}$. Any idle period lying in $R_{2}$, on the other hand (e.g., idle period $Z$ in Figure 2(b)), starts later than the job's ready time but is large enough for it. Hence, the job may be assigned to any idle period in $R_{2}$ at the cost of delaying its execution beyond its ready time.

\section{A. Partitioning of the Idle Periods}

Our objective is to obtain efficient algorithms for the online scheduling problem with general deadlines. We note that the work in [23] was developed for the special case of immediate deadlines. Recall also that the algorithm developed in [23] maintains a single priority search tree that contains all $K$ points in the plane, i.e., all $K$ idle periods on all servers. A single tree structure is appropriate for immediate deadlines, in which case each job is represented by a single point in the plane. However, it cannot be directly applied to the more general problem we are considering, in which jobs are represented by a line segment, such as the one between points 
$P$ and $P^{\prime}$ in Figure 2(b). With a single tree structure, the only way to handle a job with a general deadline is to perform multiple searches for multiple points along the line segment representing this job. Such an approach is inefficient if the points on the line segment are selected close to each other, since each search takes $O(\log K)$ time; whereas it may fail to find feasible idle periods if the points are selected far from each other to lower the worst-case running time.

In order to obtain efficient scheduling algorithms for the problem at hand, we partition the area of the plane above the diagonal into strips of width equal to twice the minimum job size $l_{\min }$. Figure 2(b) shows the partitioning of the plane into horizontal strips. Alternatively, one might partition the plane into vertical strips of width $2 \times l_{\min }$; the choice of direction depends on the optimization strategy selected, as we discuss shortly. Doing so in effect partitions the set of $K$ idle periods into a number $H$ of subsets, where subset $h, h=1, \cdots, H$, contains the idle periods falling within the $h$-th strip.

Rather than maintaining a single tree data structure as in [23], we maintain $H$ priority search trees, one for each strip. We also ignore (i.e., do not keep any information about) any idle period of length less than $l_{m i n}$, as it cannot be used for scheduling any job. Maintaining one tree structure for each strip is based on the observation that a given strip may contain at most one idle period from each server. To see that this is true, note that two consecutive idle periods on the same server must be separated by a job of length at least $l_{\text {min }}$, and that the length of each idle period is at least $l_{\min }$ (otherwise the idle period is discarded); therefore, the starting (and ending) times of two idle periods on any given server are at least $2 \times l_{\text {min }}$ time units apart from each other. In other words, the number of idle periods in a strip is bounded above by the number $n$ of servers. Consequently, updating the schedule (i.e., adding or removing idle periods) takes time $O(\log n)$, rather than $O(\log K)$, where typically $n \ll K$.

Since each priority search tree structure contains only a subset of the set of idle periods, it may be necessary to search several trees to find a feasible idle period for a new job request ${ }^{2}$. Consider point $P$ in Figure 2(b), representing the earliest time the new job may start execution. In this example, the new job can be scheduled either in the idle period represented by point $V$ or the one represented by $Y$. Point $V$ can be found by searching the tree structure corresponding to the strip in which point $P$ lies; however, if point $V$ (i.e., the corresponding idle period) did not exist, one would have to continue searching strips above the one in which $P$ lies (i.e., those with starting times earlier than the new job) in order to find an idle period (in this case, point $Y$ ) that would not delay the start of the job. On the other hand, if neither $V$ or $Y$ existed, the search would have to continue in strips below

\footnotetext{
${ }^{2}$ To improve the scalability of the algorithm, in terms of both running time and memory usage, we may partition the plane in strips of length $M \times 2 \times$ $l_{\text {min }}$, where $M$ is an integer greater than one. In this case, there will be no more than $M$ idle periods from each server within each strip, or no more than $n M$ idle periods in all. Consequently, the complexity of searching each tree becomes $O(\log (n M))$, or $O(\log M+\log n)$, but the number of strips (and corresponding trees) to be maintained decreases to $H / M$, where $H$ is the number of strips for $M=1$. Letting $M=n^{k}$, where $k$ is a small integer, reduces the number of trees by a factor of $n^{k}$ compared to the case $M=1$, while the time to search each tree increases only by a factor of $k+1$, i.e., becomes $O((k+1) \log n)$.
}

the one in which $P$ lies, to identify idle periods (e.g., $Z$ ) that could accommodate this job at some starting time along the line segment from $P$ to $P^{\prime}$.

In addition to allowing the scheduler to handle jobs with general deadlines efficiently, the partition of idle periods into subsets also enables the natural implementation of a variety of strategies for selecting one among multiple feasible idle periods. This unique feature of our approach, due to its inherent flexibility in terms of partitioning the plane either horizontally or vertically, and in terms of the order in which the strips are searched, is discussed in detail in the next subsection.

\section{B. Scheduling Strategies}

We now describe a suite of scheduling strategies which make use of the approach we outlined in the previous subsection. These strategies are based on the observation that a job scheduled in an idle period will create at most two new idle periods: one between the start of the original idle period and the start of the job (the leading idle period), and one between the end of the job and the end of the original idle period (the trailing idle period). The creation of these new, smaller idle periods results in further fragmentation of the available capacity, and may prevent future job requests from being accommodated. Therefore, it may be desirable to schedule a new job within the idle period such that the size of either the leading or trailing idle periods created is optimized, since doing so is likely to increase the chances that future jobs will fit in these new idle periods.

To illustrate how the partitioning of the plane into strips can facilitate the implementation of such scheduling strategies, consider again the new job in Figure 2. This job can be accommodated by three idle periods, corresponding to points $Y, V$, and $Z$. Selecting either point $V$ or point $Z$ will result in a leading idle period of zero length (in fact, any point in the feasible region $R_{2}$ will have the same effect). On the other hand, selecting point $Y$ in region $R_{1}$ will result in a leading idle period of length $\left(t_{6}-t_{5}\right)$; furthermore, the higher up in region $R_{1}$ a point lies, the larger the leading period that will be created if the job is assigned to it. Based on these observations, if the objective is to minimize the leading idle period, the search must start in strips within region $R_{2}$ first; if that fails, the search should continue with the bottom strip within region $R_{1}$, and proceed upwards until a feasible idle period is found. If, however, the objective is to maximize the leading idle period, then the search must start at the topmost strip of region $R_{1}$, and proceed downwards. Note also that while all points in region $R_{2}$ will result in a leading period of zero length, the later the starting time of a point the longer the execution of the new job will be delayed. This suggests that the strips of region $R_{2}$ should be searched from top to bottom to minimize the job turnaround time.

Similar observations can be made regarding the goal of optimizing the length of the trailing idle period created when scheduling a new job. This objective can be achieved by partitioning the plane in vertical strips (as opposed to the horizontal ones shown in Figure 2(b)), and following a similar search strategy.

The following strategies for the scheduling problem with general job deadlines arise naturally within this framework: 
1) Min-LIP, which minimizes the leading idle period;

2) Min-TIP, which minimizes the trailing idle period;

3) First-fit, which returns the first (i.e., earliest) feasible idle period, regardless of the sizes of the leading and trailing idle periods.

We discuss the implementation of these strategies in the next section. We have also considered the maximization versions of the first two strategies (i.e., max-LIP and max-TIP), but due to space constraints we do not discuss them here. We also note that another related strategy, best-fit, would minimize the sum of the leading and trailing idle periods. We have determined that the implementation of best-fit is significantly different than that of the three strategies listed above, in that it involves more complex, two-dimensional balanced tree structures. The best-fit strategy is the subject of ongoing research within our group, and we plan to report our results in a separate submission in the near future.

\section{Algorithm Description And Implementation}

We now describe in detail the algorithm and related balanced tree data structure for implementing the min-LIP scheduling strategy, and we analyze its worst-case running time. At the end of the section, we discuss the modifications required to implement the min-TIP and first-fit strategies.

\section{A. Balanced Tree Structure for the Min-LIP Strategy}

Recall from Section III-A that we partition the set of idle periods on all servers into $H$ subsets, each subset corresponding to one of the horizontal strips in the geometric representation of the schedule of advance reservations (refer to Figure 2(b)) and consisting of the idle periods in this strip. Each subset is of size at most $n$, where $n$ is the number of servers. The number $H$ of subsets (equivalently, of horizontal strips) depends on how far in the future users are allowed to make advance reservations. For a given system, the value of $H$ is fixed.

By construction, each subset $h, h=1, \cdots, H$, contains all idle periods with starting times in the interval $[2(h-$ 1) $\left.l_{\text {min }}, 2 h l_{\text {min }}\right)$. The idle periods in subset $h$ are stored in a priority balanced search tree $T_{h}$; in our implementation, we use augmented red-black trees [8]. Whenever the scheduling algorithm (described in the next subsection) needs to search subset $h$ to find an idle period for a new job, tree $T_{h}$ is searched; as we explain shortly, the manner in which the tree is searched depends on the part of the feasible region $\left(R_{1}\right.$ or $R_{2}$ in Figure 2(b)) in which the corresponding strip lies. The search of tree $T_{h}$ will be unsuccessful if and only if no feasible idle period for the new job exists in this strip. Otherwise, the search will return a feasible idle period that optimizes a given objective; for the min-LIP strategy we are considering, it will return the idle period that will result in the minimum leading idle period among all feasible idle periods in the strip.

In tree $T_{h}$, the actual idle periods are in the leaf nodes, arranged in ascending order of their starting time. For the minLIP strategy, a leaf node corresponding to idle period $X$ stores the following information:

- the starting time of $X$;

- the ending time of $X$; and

- other auxiliary data, such as the identity of the corresponding server.

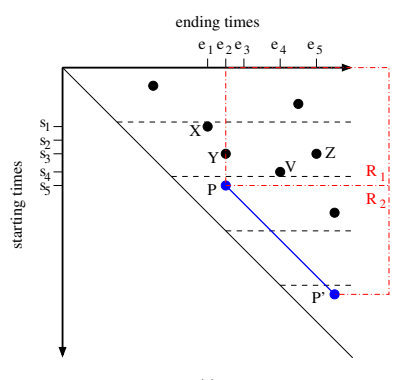

(a)

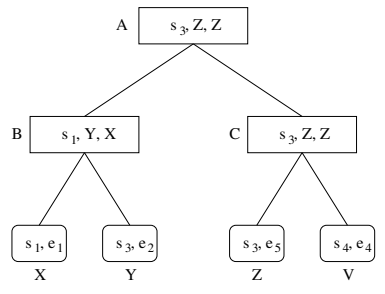

(b)
Fig. 3. (a) Schedule of advance reservations, (b) balanced tree structure storing the idle periods in the second strip from the top

Internal tree nodes store information regarding the idle periods in their subtree. This information is used to navigate the tree and locate idle periods appropriate for the new job to be scheduled. In the case of the min-LIP strategy, the information stored in internal node $v$ consists of:

- the median of the starting times of the idle periods stored in the subtree of $T_{h}$ rooted at $v$;

- a pointer to the idle period in $v$ 's subtree with the latest ending time; and

- a pointer to the idle period in $v$ 's subtree with the maximum length.

Figure 3(b) shows the balanced tree $T_{h}$ associated with the second strip from the top of the schedule shown in Figure 3(a). This strip contains four idle periods with starting and ending times: $X=\left(s_{1}, e_{1}\right), Y=\left(s_{3}, e_{2}\right), Z=\left(s_{3}, e_{5}\right)$, and $V=$ $\left(s_{4}, e_{4}\right)$. Since $s_{1}<s_{3}<s_{4}$, the idle periods are stored in this order as the leaves of the tree in Figure 3(b). Internal node $B$ of the tree stores the median $s_{1}$ of the starting times of idle periods $X$ and $Y$ stored in its subtree, along with pointers to the idle period with the latest ending time (i.e., $Y$ ) and the largest one (i.e, $X$ ); similar information is stored in node $C$ and the root $A$ of the tree.

Note that as time advances, idle periods expire (i.e., their ending time passes) and must be discarded. Our approach of partitioning the plane into strips and maintaining a separate tree structure for the idle periods within each strip makes it easy to handle expired idle periods. Let us assume that the system starts operation at time $t=0$, and that we maintain $H$ strips, each of width $2 l_{\min }$. Since the scheduling horizon (i.e., the time in the future during which a job can be scheduled) is $H \times 2 \times l_{\min }$ time units, then no idle period can end at time $t^{\prime}>t+H \times 2 \times l_{\text {min }}$, where $t$ is the current time. Consider the topmost strip with index $h=1$. Initially, the latest time at which an idle period in this strip may end (expire) is at time $t^{\prime}=(H+1) \times 2 \times l_{\text {min }}-\epsilon$, corresponding to the scheduling, at time $t=2 \times l_{\text {min }}-\epsilon$, of a job with ready time $H \times 2 \times l_{\text {min }}$ time units in the future. Therefore, at time $t=(H+1) \times 2 \times l_{\text {min }}$, the tree corresponding to strip with index $h=1$ is discarded, since all idle periods recorded in that tree have already expired. At the same time, all strips (and corresponding trees) with indices $h, h=2, \cdots, H$, are renumbered to $h^{\prime}=h-1$, and a new empty tree is created to record idle periods falling in the new strip with index $h^{\prime}=H$. This discard operation is repeated every $2 l_{\text {min }}$ time units thereafter. All the operations involved in discarding a tree can be performed in $O(1)$ time with no 
extra memory cost by using (1) a circular queue to record the tree indices, and (2) modulo- $H$ arithmetic. If a single tree structure were used instead to store all idle periods, deleting expired idle times would require additional information to be kept at internal nodes, as well as costly periodic operations to locate all idle times with past ending times.

\section{B. Min-LIP Algorithm}

Consider a request to schedule a new job $j$ with parameters $\left(r_{j}, l_{j}, d_{j}\right)$. Let $P$ and $P^{\prime}$ be the points in the geometric representation of the schedule that correspond to the earliest and latest times, respectively, at which the new job can be scheduled (refer also to Figure 3(a)). Let $p, 1 \leq p \leq H$, be the index of the horizontal strip in which point $P$ lies; let $p^{\prime} \geq p$ be the index of the strip where point $P^{\prime}$ lies. Similar to our earlier discussion, we also let $R_{1}$ (respectively, $R_{2}$ ) denote the part of the feasible region for the new job $j$ containing idle periods with starting times earlier (respectively, later) than the job's ready time $r_{j}$.

The min-LIP algorithm to find a feasible idle period for the new job $j$ that minimizes the length of the leading idle period created consists of two steps: a search in region $R_{2}$, followed by a search in region $R_{1}$, if necessary. Next, we describe these two steps in detail.

Step 1: Search in region $R_{2}$. The algorithm first searches for a feasible idle period in region $R_{2}$. Any such idle period has starting time $s \geq r_{j}$; hence, we schedule job $j$ to start at time $s$, avoiding the creation of a leading idle period. Although any feasible idle period in this region is optimal in terms of the objective we consider, assigning the new job to an idle period with starting time $s$ will delay the execution of the job by an amount of time equal to $s-r_{j}$ units beyond its ready time. In order to minimize this delay, the min-LIP algorithm explores the horizontal strips in this region in top-to-bottom fashion, i.e., by examining the corresponding trees in the order $T_{p}, T_{p+1}, \cdots, T_{p^{\prime}}$.

The min-LIP algorithm exploits the observation that any feasible idle period in region $R_{1}$ is optimal in order to examine each tree $T_{h}, h=p, \cdots, p^{\prime}$, in this region in $O(1)$ time. Recall that the root of $T_{h}$ maintains a pointer to the largest idle period in the tree (refer to Figure $3(\mathrm{~b})$ ). If this idle period is smaller than the new job, then we know that no idle period in this tree can accommodate this job, and the algorithm proceeds to examine the next tree in the region; otherwise, the algorithm assigns the job to this largest idle period. Consequently, each horizontal strip that contains no feasible idle period is eliminated in $O(1)$ time. At most one strip with a feasible idle period (the first such strip in the sequence) is examined, and the assignment of a job to the largest idle period in this strip takes time $O(1)$. In this case, the corresponding tree $T_{h}$ must also be updated (to delete the largest idle period); this operation takes $O(\log n)$ time, where $n$ is the number of servers in the system. If a trailing idle period that is larger than the minimum job size $l_{\text {min }}$ is created, it has to be inserted in the appropriate tree (which may be different than $T_{h}$ ). Locating the appropriate tree from the trailing idle period's starting time takes constant time, and the insert operation takes $O(\log n)$ time. Since the number of strips that fall within region $R_{2}$ is at most $k=\left\lceil\frac{d_{j}}{2 l_{\min }}\right\rceil$, where $d_{j}$ is the deadline of the new job, the worst-case running time of this step is $O(k+\log n)$ if the region contains a feasible idle period, and $O(k)$ if it does not.

Step 2: Search in region $R_{1}$. If Step 1 fails (i.e., no feasible idle period for the new job exists in region $R_{2}$ ), the algorithm proceeds to explore region $R_{1}$. If any feasible period in this region starting at time $s$ is selected, the job will start execution at its ready time $r_{j}$, creating a leading idle period of length $r_{j}-s$. Since our goal is to minimize this length, the algorithm examines the horizontal strips in this region in bottom-to-top fashion, i.e., it searches the corresponding trees in the order $T_{p-1}, T_{p-2}, \cdots, T_{1}$. Note also that in this step of the algorithm we may safely ignore the line segment representing the job (e.g., the segment from point $P$ to point $P^{\prime}$ in Figure 3(a)), and simply focus on the single point representing the job starting at its ready time (i.e., point $P$ ).

Each tree $T_{h}, h=p-1, \cdots, 1$, in region $R_{1}$ is searched using a standard algorithm for red-black trees [8] to find the idle period (if any) with the latest starting time that is large enough to accommodate the new job. This search takes time $O(\log n)$. If a feasible idle period is found in some tree $T_{h}$, at most three update operations must be performed: to delete the idle period from $T_{h}$, and to insert the newly created leading and trailing idle periods (as long as they are larger than $l_{\text {min }}$ ) into the appropriate trees; all these operations take $O(\log n)$ time [8], [10]. The number of strips within region $R_{1}$ is at most $m=\left\lceil\frac{r_{j}}{2 l_{m i n}}\right\rceil$, where $r_{j}$ is the ready time of job $j$. The worst-case running time of this step is $O(m \log n)$ and occurs when either no feasible idle period exists, or one exists in the topmost strip. Similarly, the worst-case running time of the overall algorithm is $O(k+m \log n)$.

Let us illustrate how the tree search algorithm operates by considering the second strip from the top in Figure 3(a), i.e., the one containing the idle periods $X, Y, Z$, and $V$. It is clear from the figure that only $Y, Z$, and $V$ can accommodate the new job; of these, $V$ is optimal in terms of minimizing the leading idle period for the job represented by point $P$, as it has the latest starting time.

The algorithm starts at the root $A$ of the tree in Figure 3(b) that stores the idle periods in this strip. It compares the ready time $\left(r_{j}=s_{5}\right)$ of the new job $j$ to the median $\left(=s_{3}\right)$ of the starting times of the idle periods in this tree stored at the root. In this case, $s_{3}<s_{5}$, which implies that some idle periods in the left subtree of $A$, as well as some idle periods in the right subtree, start before $r_{j}$, hence both subtrees may have to be examined further (if the reverse were true, the algorithm would have eliminated the right subtree of $A$ immediately). The algorithm then compares the ending time of the job $\left(=e_{2}\right)$ to the maximum ending time of the idle periods in the left subtree of $A$; this value $\left(=e_{2}\right)$ can be obtained by following the pointer to the idle period $Y$ with the maximum ending time that is stored in the root $B$ of the left subtree. Since the two values are equal, a feasible idle period may exist for this job in the subtree rooted in $B$. Therefore, the algorithm marks node $B$ for possible consideration in the future, and proceeds to examine the right subtree of $A$.

The search continues in a recursive manner until a leaf node is reached. In this example, the ready time $\left(r_{j}=s_{5}\right)$ of the job is compared to the median starting time $s_{3}$ stored in node 
$C$. Since $s_{3}<s_{5}$, the algorithm compares the ending time ( $=e_{5}$ ) of the left child of $C$ to the ending time $e_{2}$ of the job. Since $e_{5}>e_{2}$, the idle period $Z$ in the left child of $C$ is feasible, and the algorithm marks the leaf node $Z$. It then similarly examines the right child of $C$, and determines that it also represents a feasible idle period; since this is the one with the latest starting time, it is optimal and is the one returned by the algorithm. In general, once the algorithm reaches a leaf node, all idle periods with starting time earlier than or equal to $r_{j}$ are to its left. If the idle period represented by this leaf is feasible, then it is returned and the algorithm terminates. Otherwise, it is sufficient to continue the search recursively from the last marked node. Due to space constraints, we are unable to include a formal description of the algorithm.

\section{Implementation of Other Scheduling Strategies}

The scheduling strategies we defined in Section III-B can be implemented by appropriately modifying either the tree data structure or the search algorithm we described above for the min-LIP strategy. In order to optimize the trailing idle period, the plane must be partitioned into vertical strips of length $M \times$ $2 \times l_{\text {min }}, M \geq 1$, and each tree must store the idle periods in the corresponding strip in increasing order of their ending, rather than starting, times; the search algorithm is similar to the corresponding algorithm for min-TIP. Finally, the first fit strategy can be implemented by exploring the horizontal strips in increasing order of index $h$, and selecting from each tree the first feasible idle period found.

\section{Performance Evaluation}

We use simulation to evaluate the performance of the various scheduling strategies. We use the method of batch means to estimate the performance parameters we consider (and which we discuss shortly), with each batch consisting of thirty simulation runs and each run lasting until $10^{6}$ jobs have been submitted to the Grid scheduler. We have also obtained $95 \%$ confidence intervals for all the results, which are shown in the figures.

In our simulation, we assume that job requests arrive as a Poisson process with rate $\lambda$. Job sizes are distributed according to a bounded Pareto distribution. The minimum job size is set equal to 1 , and is taken as the unit of time. The maximum job size is set to 50 time units, and we vary the mean job size $\bar{x}$ by changing the value of the parameters of the Pareto distribution. We let $L$ denote the amount of time that the scheduler $\mathcal{S}$ can look "into the future"; in other words, a job may request to be scheduled at most $L$ units of time in the future. We let the deadline $d_{j}$ of job $j$ be uniformly distributed in the interval $\left(r_{j}+l_{j}, r_{j}+l_{j}+q\left(L-r_{j}-l_{j}\right)\right)$, where $q, 0 \leq q \leq 1$ is a parameter that controls the "tightness" of the job deadlines. In our simulations, we let $L=200$.

We use four performance metrics in our study. The loss rate is the fraction of jobs that are dropped due to the fact that their deadline cannot be met. The system utilization is the fraction of time the $n$ servers are busy serving jobs. The average delay is the mean amount of time that a job has to wait beyond its ready time until it starts execution; note that dropped jobs do not contribute to the average delay. Finally, the fairness ratio is a measure of how fairly jobs of different sizes fare in terms of drop probability under a given scheduling algorithm. To compute the fairness index, we partition the domain $[1,50]$ of the job size distribution into $B=100$ bins of equal size. Let $z_{i}$ be the number of arriving jobs that fall into the $i$-th bin during a certain simulation run, and let $z_{i}^{\prime} \leq z_{i}$ be the number of these jobs that are scheduled successfully. Let $w_{i}$ be the fraction of jobs in the Pareto distribution that fall in the $i$-th bin. The fairness index $F$ of a scheduling strategy is calculated as:

$$
0 \leq F=\sum_{i=1}^{B} w_{i} \frac{z_{i}^{\prime}}{z_{i}} \leq 1 .
$$

Clearly, the closer the value of the fairness index is to one, the more fair the scheduling discipline is.

We compare four scheduling strategies: first-fit, min-LIP, min-TIP, and LACT. The LACT algorithm, which we described in Section II, does not consider the idle periods created at each server, and hence suffers the effects of capacity fragmentation; we consider this algorithm as a baseline case. Although we do not show any results for the max-LIP and max-TIP scheduling algorithms, their overall behavior is similar to that of min-LIP and min-TIP in that they are efficient in utilizing the available system capacity.

Figures 4-7 plot the loss rate, utilization, average delay, and fairness ratio, respectively, for the four scheduling strategies against the system load $\rho$. The system load is calculated using the familiar from queueing theory expression $\rho=(\lambda \bar{x}) / n$. For the results shown in these figures, we let the number of servers $n=20$, the mean job size $\bar{x}=3.28$, and the tightness of the job deadlines $q=0.1$. Note that the load values in the figures range from low $(\rho=0.1)$ to very high $(\rho=1.1)$ at which the system is more than $100 \%$ loaded. Also, the $95 \%$ confidence intervals are quite narrow for all curves shown.

From Figure 4 we can see that the loss rate increases with the system load for all four scheduling algorithms, as expected. However, the LACT algorithm performs significantly worse than the other three strategies at all but very low loads; this result is not surprising given the fact that this algorithm does not consider the idle periods in the servers. Under the other three strategies, jobs experience low loss rates even for load values close to 1 ; in fact, min-LIP and min-TIP have almost identical behavior with loss rates close to zero for loads up to $\rho=0.8$. The first-fit algorithm also experiences low loss, but it performs worse than min-LIP or min-TIP for all load values less than 1. Therefore, min-LIP and min-TIP are clearly the best algorithms for typical operating regimes (i.e., at medium to medium-high loads). Note also that the loss rate for two algorithms is less than $10 \%$ even at a load of $\rho=1.1$. This result can be explained by the fact that when the system is overloaded, large jobs have higher probability to be dropped than small jobs, under these two algorithms; hence at $\rho=1.1$, the dropped jobs account for more than $10 \%$ of the offered load.

Figure 5, which plots the system utilization versus the load, confirms our observations regarding the relative performance of the four algorithms. As expected, utilization increases with the system load initially, but at some point the curves level off. LACT shows the lowest utilization, a result consistent with the high loss rates we observed in Figure 4. Min-LIP 


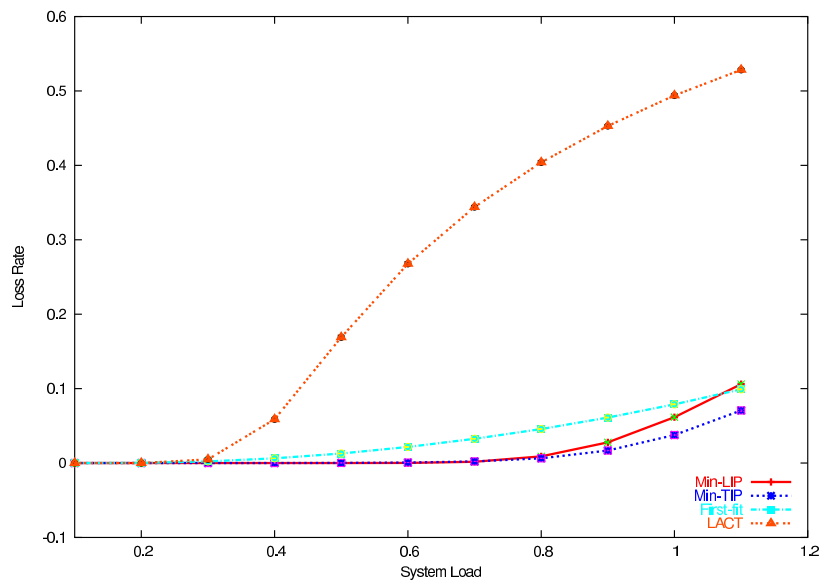

Fig. 4. Loss rate vs. system load $\rho, n=20, \bar{x}=3.28, q=0.1$

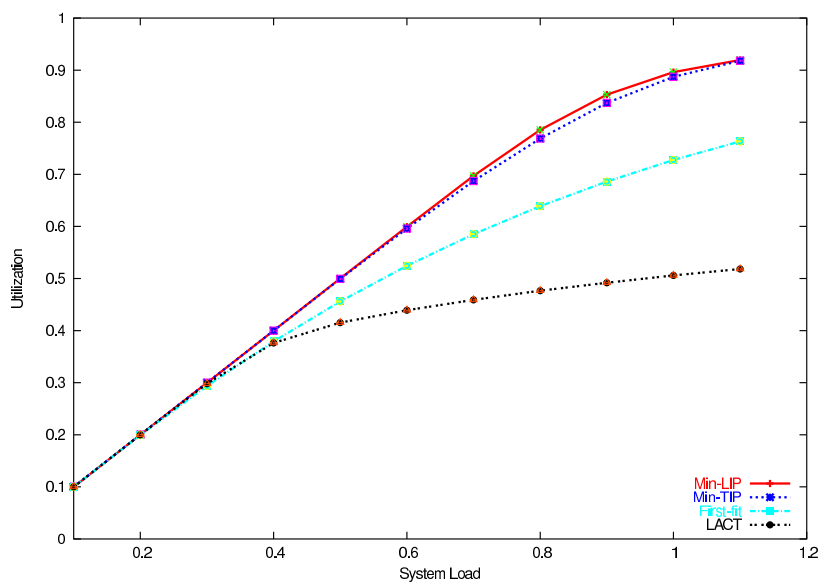

Fig. 5. Utilization vs. system load $\rho, n=20, \bar{x}=3.28, q=0.1$

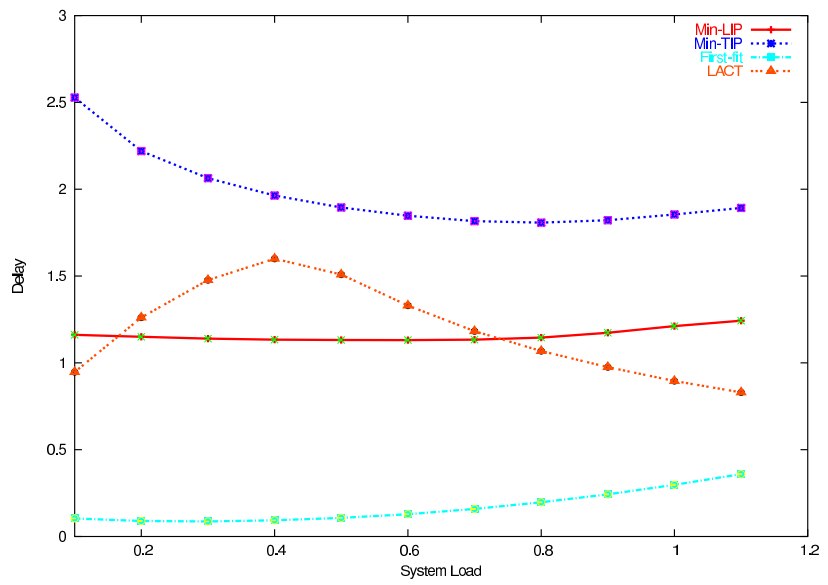

Fig. 6. Average delay vs. system load $\rho, n=20, \bar{x}=3.28, q=0.1$ and min-TIP again have the best performance, followed by first-fit. Moreover, the behavior of the min-LIP and min-TIP curves is almost identical, with utilization increasing almost linearly with the load values. This result indicates that both algorithms are capable of identifying and using idle periods to schedule jobs, thus ensuring that fragmentation of system capacity does not compromise overall performance. We also note that the difference in utilization between first-fit, on the one hand, and min-LIP and min-TIP, on the other hand, is higher than the difference in loss rates would suggest. The higher difference in utilization can be explained by the fact that the first-fit strategy is less fair than the other two, and tends to drop larger jobs with higher probability; we will discuss this fairness issue in more detail shortly.

Let us now turn to Figure 6 which plots the average job delay against the system load. As we can see, jobs experience the lowest delay under the first-fit strategy. This result agrees with intuition: first-fit assigns a new job to the earliest feasible idle period, thus minimizing delay. We also observe that the average delay for min-LIP is higher than for first-fit but lower than under min-TIP. Recall that min-LIP first searches for the earliest feasible idle period in region $R_{2}$ (i.e., for an idle period starting after the job's ready time). Once such an idle period is found, the job is scheduled to start at the beginning of this period. Consequently, the starting time of the job can be no earlier than under first-fit, hence the longer delay. On the other hand, min-TIP also searches first for the earliest idle period starting after a job's ready time. But unlike min-LIP, it schedules the job at the end of this idle period; shifting the job so that its completion time coincides with the end of the idle period causes higher delay than min-LIP. The average delay curve for the LACT algorithm lies between the corresponding curves for min-LIP and min-TIP for most system load values of interest. Note that the average delay for LACT increases up to $\rho=0.4$, at which point LACT losses start to accelerate (refer to Figure 4). Beyond that point, average delay under LACT starts to decrease; however, this behavior is a side effect of the high losses incurred, rather than an indication of an inherent quality of the algorithm.

Overall, the average delay values in Figure 6 are relatively low, and correspond to a fraction of the mean job size $\bar{x}=3.28$ for all algorithms. More importantly, average delay for the three strategies of interest (i.e., first-fit, min-LIP, and min-TIP) does not vary significantly with load, although it increases slightly at high loads. One exception is the min-TIP strategy which shows a moderate decrease in delay as $\rho$ increases from low to moderate values. This behavior can be explained as follows. At low loads, min-TIP can find feasible idle periods starting after the jobs' ready time, and shifts the jobs to the end of these idle periods incurring a relatively high delay. At higher loads, on the other hand, and due to the relatively tight deadlines, it becomes more difficult to find such idle periods. In case of failure, min-TIP (similar to min-LIP) then searches for feasible idle periods that start before the jobs' ready time. Since these idle periods start earlier, the average delay under min-TIP tends to decrease with the load.

Figure 7 plots the fairness index, calculated by expression (1), against the system load. As we can see, the fairness index of the LACT algorithm suffers a precipitous drop start- 


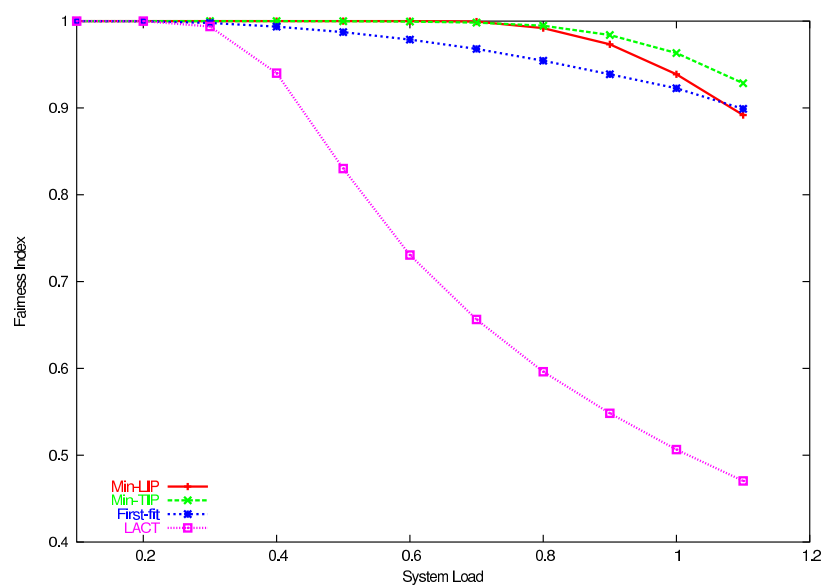

Fig. 7. Fairness ratio vs. system load $\rho, n=20, \bar{x}=3.28, q=0.1$

ing at $\rho=0.4$, the point where its losses begin to accelerate. This increase in unfairness is primarily due to the fact that larger jobs experience a significantly larger drop probability than smaller ones. The first-fit strategy is more fair than LACT, but it starts being unfair to jobs of larger size at loads as low as $\rho=0.4$, compared to min-LIP and min-TIP; as a result, its utilization of the system capacity starts suffering from that point, as illustrated in Figure 5. The min-LIP and min-TIP strategies, on the other hand, achieve fairness index values close to 1 even at high system loads, with min-TIP slightly outperforming min-LIP. The fact that min-LIP and min-TIP remain fair across a wide range of load values is an important property of these algorithms, and indicates that they are capable of exploiting the idle periods in an effective manner. Moreover, their ability to treat all jobs fairly implies that users will not need to employ strategies such as splitting a large job into several smaller ones, to avoid discrimination. Note that such strategies impose an additional overhead to the system in the form of additional memory usage (needed to store the additional idle periods created) and higher running time (due to the larger number of jobs requests, each request needing to search a larger data structure).

In addition to providing insight into the relative behavior of the four strategies due to the different optimization objectives considered, Figures 4-7 illustrate that properly designed scheduling algorithms can effectively overcome the obstacles of capacity fragmentation to deliver high performance in terms of metrics that reflect the requirements of both users and service providers. Specifically, the min-LIP and minTIP algorithms cater to the user needs by ensuring that job deadlines are met in a fair manner while keeping both loss rates and average delay low; at the same time, they deliver high system utilization, an important goal for service providers.

The next three Figures 8-9 illustrate the behavior of the loss rate as we vary the values of three important system parameters, namely, mean job size $\bar{x}$, deadline tightness $q$, and number of servers $n$, respectively; the other parameters in the experiments take values as specified in the corresponding figure caption. Due to space constraints, we are unable to present results for the other three performance metrics; however, when appropriate, in our discussion below we address the effect of the various system parameters on these metrics.

Figure 8 plots the loss rate for the four scheduling algorithms against the mean job size for $n=20$ servers and system load $\rho=0.6$. Min-LIP and min-TIP clearly outperform the other two algorithms, and their loss rate remains well below $1 \%$ across the range of mean job size values shown in the figure. In fact, mean job size has little effect on the loss rate for these algorithms. We have also found that utilization remains close to $60 \%$ for these two algorithms, and the fairness index close to 1. First-fit has a higher loss rate, which increases with the mean job size. Furthermore, we have found that the unfairness of first-fit also increases with the mean job size, to the degree that system utilization drops much more than the loss rate suggests, and in fact, it drops below the utilization of the LACT algorithm for $\bar{x}>6$. Finally, the loss rate of LACT is the highest, but it decreases as $\bar{x}$ increases. While this behavior may seem counter-intuitive, it can be explained by noting that for constant load, increasing $\bar{x}$ implies a lower job arrival rate. Fewer job arrivals result in fewer idle periods, hence a lower degree of fragmentation of the available capacity. Since LACT performs worse with increasing degree of fragmentation, its performance improves as the mean job size increases.

In Figure 9 we plot the loss rate against the deadline tightness $q$. Recall that the larger the value of parameter $q$, the further in the future the deadline of each job lies, and the more flexibility an algorithm has in scheduling jobs. As we can see in the figure, the loss rate of the min-LIP and minTIP strategies decreases as the value of $q$ increases from 0 (the case of immediate deadlines) to 0.1 ; after that point, the loss rate remains at zero. The loss rate of first-fit also decreases initially, and then remains low throughout the range of values of $q$. This behavior indicates that these three policies, which consider the idle periods when scheduling jobs, are effective throughout the range of deadlines considered in our study; their performance is affected, although not significantly, only when deadlines are very "tight." On the other hand, it is evident that the LACT algorithm is very sensitive to the tightness of the deadlines: its performance is poor when $q$ is small, but it improves dramatically as the value of $q$ increases, in which case the algorithm can push the starting time of jobs further in the future without missing their deadlines. Of course, this improvement in performance comes at the expense of significantly higher delay (not shown here due to space constraints).

Finally, Figure 10 plots the loss rate against the number $n$ of servers in the Grid. The relative behavior of the various curves is similar to the one observed earlier: min-LIP and min-TIP clearly outperform the other two strategies and have loss rates close to zero at larger values of $n$, while LACT has by far the worse performance. In general, the loss rate decreases with the number of servers for all strategies, but shows a significant improvement for LACT. This behavior can be explained by noting that at constant load, as the number of servers increases, the degree of fragmentation tends to decrease, hence the performance of LACT improves. We also emphasize that the loss rate for LACT is an order of magnitude higher than the loss rates of either min-LIP or min-TIP throughout the values 
of $n$ used in this experiment.

\section{CONCLUding REMARKS}

We have applied techniques from computational geometry to develop a suite of scheduling strategies that allocate resources in a Grid environment using a range of optimization criteria. We also presented efficient implementation of the various algorithms that scale to large Grid systems. We have presented results from extensive simulation experiments to demonstrate that our algorithms are simultaneously userand system-centric: they are able to schedule resources to meet the deadlines imposed by users and maximize system utilization, while experiencing low job drop rates and low delays. Our algorithms also allocate resources to users in a fair manner. Our work provides a practical and efficient solution to the problem of scheduling resources in the emerging highly dynamic Grid environments.

\section{REFERENCES}

[1] R. J. Al-Ali, et al. Analysis and provision of QoS for distributed grid applications. Journal of Grid Computing, 2(2):163-182, June 2004.

[2] B. Bode, et al. The portable batch scheduler and the Maui scheduler on linux clusters. In Proceedings of the Usenix Conference, 2000.

[3] R. Buyya, D. Abramson, and S. Venugopal. The grid economy. Proceedings of the IEEE, 93(3):698-714, March 2005.

[4] R. Buyya, D.Abramson, and J. Giddy. Nimrod/g: An architecture for a resource management and scheduling system in a global computational grid. In Proceedings of HPC'O0-Asia, pages 283-289, May 2000.

[5] E-K. Byun, J-W. Jang, W. Jung, and J-S. Kim. A dynamic Grid services deployment mechanism for on-demand resource provisioning. In Proceedings of Cluster Computing and the Grid, 2005.

[6] E. Caronand, P. K. Chouhan, and F. Desprez. Deadline scheduling with priority for client-server systems on the grid. IEEE/ACM International Workshop on Grid Computing, pages 410-414, 2004.

[7] H-L. Chan, et al. Nonmigratory online deadline scheduling on multiprocessors. SIAM J. Computing, 34(3):669-682, 2005.

[8] T. Cormen, C. Leiserson, R. River, and C. Stein. Introduction to Algorithms. McGraw-Hill Book Company, second edition, 2001.

[9] Platform Computing Corporation. http://www.platform.com.

[10] M. de Berg, et al. Computational Geometry: Algorithms and Applications. Springer-Verlag, second edition, 2000.

[11] E. Elmroth and J. Tordsson. A grid resource broker supporting advance reservations and benchmark-based resource selection. LNCS volume 3732, pages 1077-1085. Springer-Verlag, 2005.

[12] I. Foster and C. Kesselman, editors. The Grid 2: Blueprint for a New Computing Infrastructure. Morgan Kaufmann, 2003

[13] D. Jackson. New issues and new capabilities in HPC scheduling with the Maui scheduler. http://www.linuxclustersinstitute.org/Linux-HPCRevolution/Archive/PDF01/Jackson_Utah.pdf.

[14] D. Jackson, Q. Snell, and M. Clement. Core algorithms of the maui scheduler. LNCS volume 2221, pages 87-102, 2001.

[15] M. Maheswaran K. Krauter, R. Buyya. A taxonomy and survey of grid resource management systems for distributed computing. Software: Practice and Experience, 32(2):135-164, February 2002.

[16] W. Leinberger and V. Kumar. Information power grid: The new frontier in parallel computing? IEEE Concurrency, 7(4):75-84, 1999.

[17] E. McCreight. Priority search trees. SIAM Journal of Computing, 14(2):257-276, 1985.

[18] Sun Microsystems. http://www.sun.com/service/sungrid/, April 2006.

[19] R. Min and M. Maheswaran. Scheduling advance reservations with priorities in grid computing systems. In Proceedings of PDCS'01, pages 172-176, 2001 .

[20] W. Smith, I. Foster, and V. Taylor. Scheduling with advanced reservations. In Proceedings of IPDPS'00, pages 127-132, 2000.

[21] A. Sulistio and R. Buyya. A grid simulation infrastructure supporting advance reservation. In Proceedings of PDCS'04, pages 1-7, Nov. 2004.

[22] A. Takefusa, H. Casanova, S. Matsuoka, and F. Berman. A study of deadline scheduling for client-server systems on the computational grid. In Proceedings of HPDC, pages 406-415, 2001.

[23] J. Xu, C. Qiao, J. Li, and G. Xu. Efficient burst scheduling algorithms in optical burst-switched networks using geometric techniques. IEEE JSAC, 22(9):1796-1811, November 2004.



Fig. 8. Loss rate vs. mean job size $\bar{x}, n=20, \rho=0.6, q=0.1$

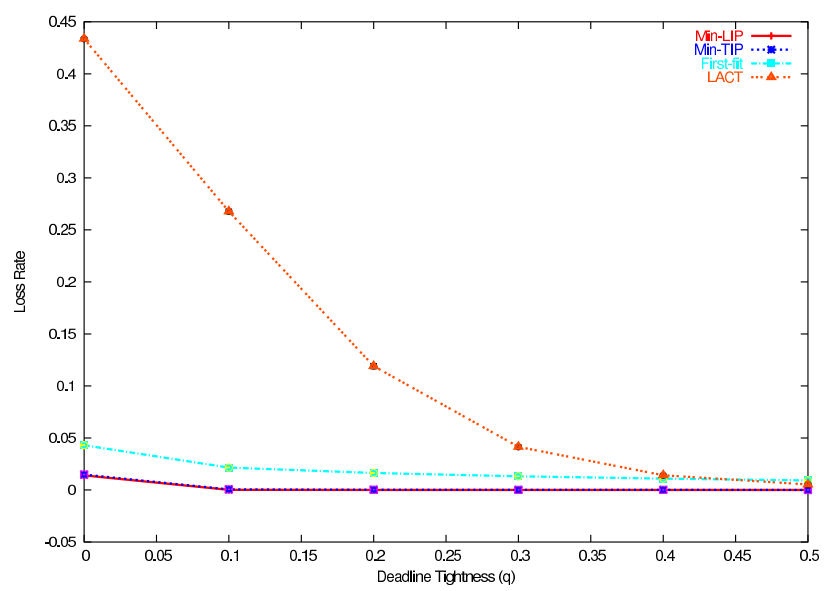

Fig. 9. Loss rate vs. deadline tightness $q, n=20, \bar{x}=3.28, \rho=0.6$

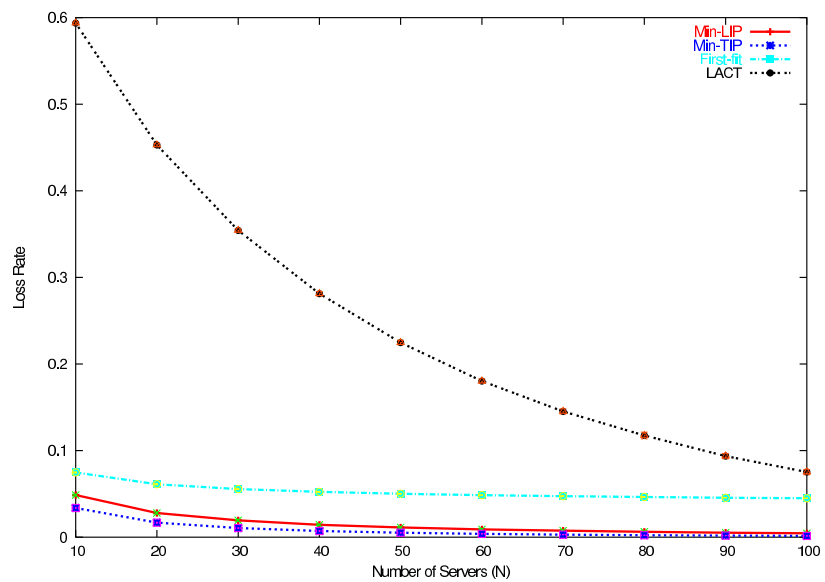

Fig. 10. Loss rate vs. number of servers $n, \bar{x}=3.28, \rho=0.9, q=0.1$ 